\title{
Retrospective Chart Review of Advanced Practice Pharmacist Prescribing of Controlled Substances for Pain Management at the Harry S. Truman Memorial Veterans' Hospital
}

\author{
Courtney Kominek, PharmD
}

\begin{abstract}
Background: The US Department of Health and Human Services' 5-point strategy to combat the opioid overdose public health crisis includes improved pain management. There is a shortage of adequately trained health care providers in pain management. Advanced practice pharmacists may be able to help fill that void. The objective of this project was to identify the impact of an advanced practice pharmacist with controlled substance prescriptive authority on morphine milligram equivalent dose (MME) and compliance with opioid risk mitigation.
\end{abstract}

Methods: In March 2020, a single-site retrospective chart review was conducted of patients who were prescribed controlled substances from July 1, 2018 to January 31, 2020. Patients received care through the outpatient Pharmacy Pain Clinic in-person or via telephone who were enrolled at the Harry S. Truman Memorial Veterans' Hospital in Columbia, Missouri, or associated outlying outpatient clinics. Patients were included if they were referred to the Pharmacy Pain Clinic and prescribed a Schedule II or III opioid medication. A 2-sided $t$ test was conducted to compare MME, and a Fisher exact test was used to compare adherence to opioid risk mitigation.

Results: Patients seen in Pharmacy Pain Clinic had a statistically significant reduction in MME from consult (93 MME) to discharge (31 MME) $(P<.01)$. There was also a statistically significant $(P<.01)$ improvement in use of opioid risk mitigation strategies, including urine drug screen, informed consent, naloxone, prescription drug monitoring program checks, and stratification tool for opioid risk mitigation dashboard reviews.

Conclusions: An advanced practice pharmacist with controlled substance prescriptive authority improved patient care with demonstrated statistically significant differences in MME and adherence with opioid risk mitigation from consult to discharge. Health care teams should look to add advanced practice pharmacists to their team as medication experts to deliver comprehensive medication management, which can include controlled substance prescribing and management.

\section{Courtney Kominek is a}

Clinical Pharmacy

Specialist-Pain

Management at the Harry

S. Truman Memorial

Veterans' Hospital in

Columbia, Missouri.

Correspondence:

Courtney Kominek

(courtney.kominek@va.gov)

Fed Pract. 2021;38(1):20-27 doi:10.12788/fp.0079 $\mathrm{n}$ the midst of an opioid overdose public health crisis, the US Department of Health and Human Services developed a 5-point strategy to combat this problem. One aspect of this strategy is improved pain management. ${ }^{1}$ There is high demand for pain management services with a limited number of health care professionals appropriately trained to deliver care. ${ }^{2}$ Pharmacists are integral members of the interdisciplinary pain team and meet this demand.

\section{BACKGROUND}

For almost 50 years, pharmacists at the US Department of Veterans Affairs (VA) have been functioning as advanced practice providers (APP) ${ }^{3}$ Clinical pharmacy specialists (CPS) provide comprehensive medication management (CMM) and have a scope of practice (SOP). The SOP serves as the collaborating agreement and outlines the clinical duties permitted in delivering patient care. In addition, the SOP may indicate specific practice areas and are standardized across VA (Table 1), ${ }^{4,5}$ Pharmacists apply for a SOP and must prove their competency in the practice area and provide documentation of their education, training, experience, knowledge, and skills. ${ }^{5,6}$ Residency and/or board certification are not required though helpful. A pharmacist's SOP is reviewed and approved by the facility executive committee. ${ }^{5}$ Pharmacists with a SOP undergo professional practice evaluation twice a year. Prescribing controlled substances is permissible in the SOP if approved by the facility and allowed by the state of licensure. According to the US Drug Enforcement Agency (DEA) as of February 10, 2020, 8 states (California, Washington, Idaho, Massachusetts, Montana, New Mexico, North Carolina, and Ohio) allow pharmacists to prescribe controlled substances. ${ }^{7}$

The VA developed the Pharmacists Achieve Results with Medications Documentation (PhARMD) tool that allows clinical pharmacists to document specific interventions made during clinical care and is included in their progress note. Data from fiscal year 2017 demonstrates that 136,041 pain management interventions were made by pharmacists across VA. 
The majority of these interventions were implemented by a CPS working autonomously as an APP. ${ }^{8}$

Several articles discuss the pharmacists role in the opioid crisis, although no outcomes data were provided. Chisholm-Burns and colleagues listed multiple potential ways that pharmacists can intervene, including managing pain in primary care clinic settings by using collaborative drug therapy agreements (CDTAs), using opioid exit plans and discharge planning in collaboration with other health care providers (HCPs), or making recommendations to the prescribers before writing prescriptions. ${ }^{9}$ Compton and colleagues similarly reviewed pharmacist roles in the opioid crisis. However, their focus was on dispensing pharmacists that provided education to patients about storage and disposal of opioids, identified opioid misuse, provided opioid overdose education and naloxone, and checked prescription drug monitoring programs (PDMPs). ${ }^{10}$ Missing from these articles was the role of the clinical pharmacist working as an APP delivering direct patient care and prescribing controlled substances.

Hammer and colleagues discussed the role of an oncology CPS with controlled substance prescriptive authority in pain management at an outpatient cancer center in Washington state. ${ }^{11}$ Under a CDTA, pharmacists could prescribe medications, including controlled substances if they obtain DEA registration. The pharmacist completed a comprehensive in-person assessment. The attending physician conducted a physical examination. Then the pharmacist presented the patient and proposed regimen to the interprofessional team to determine a final plan. Ultimately, the pharmacist wrote any controlled substance prescriptions. The patient followed up every 1 to 4 weeks by telephone with a nurse, and in-person assessments occurred at least every 6 months. No outcomes data were provided. ${ }^{11}$

Dole and colleagues reviewed the role of a pharmacist who had controlled substance prescriptive authority in a pain management clinic. The pharmacist provider saw up to 18 patients a day and then managed refill requests for 3 hours a day. The main outcome was change in visual analog scale (VAS) pain scores. Findings showed that reductions in
TABLE 1 Clinical Duties Authorized in Pharmacists Scope of Practice in the Veterans Health Administration

- Performing physical measurements and objective assessments to ensure appropriate response to drug therapy

- Ordering medications, patient care supplies, and vaccines as necessary for the provision of pharmaceutical care

- Identifying and taking specific corrective action for drug-induced problems according to protocol, procedure, guideline, or standard of care

- Ordering consults, as appropriate, to maximize positive drug therapy outcomes

- Providing clinical pharmacy expertise, comprehensive medication management, and monitoring for practice-based areas

- Obtaining and documenting informed consent for treatments and procedures for which the clinical pharmacist is responsible

- Prescribing controlled substances if authorized by the facility and state of licensure, performed in accordance with Federal regulations and Veterans Health Administration policy

VAS pain scores were statistically significant $(P<.01)$. The pharmacist processed about 150 refills with an unclear number of controlled substances requests a day based on a medication-refill protocol. This was felt to improve access to physicians for acute needs, improve consistency in refills, and capture patients in need of follow-up. Additionally, the clinic saved $\$ 455,238$ after 1 year. ${ }^{12}$

\section{Study Aims}

A review of the literature indicated sparse data on the impact of a pharmacist on opioid tapering, opioid dose, and opioid risk mitigation when the pharmacist is prescribing controlled substances. The purpose of this retrospective review was to characterize the controlled substance prescribing practices by the pharmacy pain clinic. The aim was to examine the pharmacist impact on morphine milligram equivalent (MME) and compliance with opioid risk mitigation strategies.

\section{METHODS}

This project was a retrospective, singlecenter, chart review. The project was reviewed and approved by the University of Missouri-Columbia Institutional Review Board used by the Harry S. Truman Memorial Veterans' Hospital (HSTMVH) as a quality improvement project. The author applied for controlled substance registration through the DEA and was issued registration April 30, 2018. The State of Ohio Board of Pharmacy was contacted as required by Ohio Administrative Code. The author's updated SOP to allow controlled substance prescribing 
TABLE 2 Pharmacy Pain Clinic Patient Demographics $(\mathrm{N}=75)$

\begin{tabular}{|c|c|}
\hline Characteristics & Results \\
\hline Age, mean, y & 66 \\
\hline Male sex, No. (\%) & Male $66(88)$ \\
\hline \multicolumn{2}{|l|}{ Comorbidities at consult, No. (\%) } \\
\hline Chronic obstructive pulmonary disease & $28(37)$ \\
\hline Sleep apnea & $36(48)$ \\
\hline Mental health diagnosis & $41(55)$ \\
\hline Constipation & $10(13)$ \\
\hline Overdose & $2(3)$ \\
\hline Substance use disorder ${ }^{b}$ & $10(13)$ \\
\hline \multicolumn{2}{|l|}{ Source of consult, No. (\%) } \\
\hline Primary care provider & $33(44)$ \\
\hline Physical medicine and rehabilitation & $8(11)$ \\
\hline Pain clinic & $32(43)$ \\
\hline Behavioral health performance program ${ }^{c}$ & $1(1)$ \\
\hline Self & $1(1)$ \\
\hline \multicolumn{2}{|l|}{ Reason for consult, No. $(\%)^{a}$} \\
\hline Nonopioid optimization & $14(19)$ \\
\hline Opioid monitoring or optimization & $28(37)$ \\
\hline Opioid conversion & $6(8)$ \\
\hline Opioid taper & $36(48)$ \\
\hline Performance program evaluation ${ }^{c}$ & $1(1)$ \\
\hline \multicolumn{2}{|l|}{ Pain Location/diagnosis at consult, No. $(\%)^{\mathrm{a}}$} \\
\hline Chronic low back pain & $42(56)$ \\
\hline Fibromyalgia & $6(8)$ \\
\hline Neck pain & $15(20)$ \\
\hline Spinal cord injury & $4(5)$ \\
\hline Osteoarthritis & $12(16)$ \\
\hline Peripheral neuropathy & $9(12)$ \\
\hline \multicolumn{2}{|c|}{$\begin{array}{l}\text { aMay have }>1 \text { for each patient. } \\
\text { 'Excludes tobacco use disorder; not included in mental health diagnosis. } \\
\text { "An interdisciplinary intensive pain program involving cognitive behavioral } \\
\text { therapy for chronic pain, physical therapy, occupation therapy, nutrition, } \\
\text { pharmacy, yoga or tai chi, pain management physician, and battlefield } \\
\text { acupuncture. }\end{array}$} \\
\hline
\end{tabular}

was approved July 23, 2018. The CPS functions as an APP within an interdisciplinary pain management team that includes physicians, occupational and physical therapists, complementary and integrative health, and a psychologist. The reason for Pharmacy Pain Consult is required and it is primarily submitted through the electronic health record. The consult is reviewed for appropriateness and once approved is scheduled by support staff. Once the patient is stabilized, the patient is discharged back to their primary care provider (PCP) or referring provider for continued care. Patients were considered stabilized when their patient-specific goals were met, which varied from use of the lowest effective opioid dose to taper to discontinuation of opioids with no further medication changes needed. The taper strategy for each patient was individualized. Patients were generally tapered on their existing opioid medication unless they were new to the VA and on nonformulary medications or experiencing a significant adverse reaction. $\mathrm{Nu}$ merous references are available through VA to assist with opioid tapering. ${ }^{13,14}$ The CPS is able to refer patients to other services, including behavioral health for substance use disorder treatment and medication-assisted treatment if concerns were identified.

Initial data were collected from the Veterans Integrated Service Network (VISN) 15 Corporate Data Warehouse by the VISN pharmacy analytics program manager. The original report included patients prescribed a Schedule II to V controlled substance by the author from July 1, 2018 to January 31, 2020. Chart review was conducted on each patient to obtain additional data. At the time of consult and discharge the following data were collected: opioid medication; MME; use of opioid risk mitigation strategies, such as urine drug screens (UDS), informed consent, opioid overdose education and naloxone distribution program (OEND), risk assessment via stratification tool for opioid risk mitigation (STORM), PDMP checks; and nonopioid medication number and classes.

Patients were included in the review if they were prescribed an opioid Schedule II or III controlled substance between July 1 , 2018 and January 31, 2020. Patient were excluded if they were prescribed an opioid Schedule II or III controlled substance primarily as coverage for another prescriber. Patients prescribed only pregabalin, tramadol, or a benzodiazepine also were excluded.

The primary endpoint was change in MME from baseline to discharge from clinic. Secondary endpoints included change in opioid risk mitigation strategies and change in opioid medications prescribed from baseline to discharge.

Descriptive statistics were used to analyze parts of the data. A 2-sided $t$ test was used to compare baseline and discharge MME. The Fisher exact test was used to compare nominal data of opioid risk mitigation strategies. 
Calculation of MME was performed using the conversion factors provided by the Centers Disease Control and Prevention (CDC) for opioid guideline. ${ }^{15}$ For buprenorphine, tapentadol, and levorphanol conversion ratios were obtained from other sources. The conversion ratios used, included 75:1 for oral morphine to transdermal buprenorphine, 1:3.3 for oral morphine to oral tapentadol, and 1:7.5 for oral levorphanol to oral morphine. ${ }^{16,17}$ The Revised Standards for Quality Improvement Reporting Excellence (SQUIRE 2.0) was used to write the manuscript. ${ }^{18}$

\section{RESULTS}

Seventy-five patients were included in this review. The average age of patients was 66 years; and $12 \%$ were female $(n=9)$ (Table 2). The largest number of consults came from PCPs $(44 \%, n=33)$ and the pain clinic $(43 \%$, $n=32)$. Nearly half $(48 \%)$ of the consultations were for opioid tapering $(\mathrm{n}=36)$, followed by $37 \%$ for opioid optimization or monitoring $(\mathrm{n}=28)$, and $19 \%$ for nonopioid optimization $(\mathrm{n}=14)$. The most common primary diagnoses at consultation were for chronic low back pain (56\%), chronic neck pain (20\%), and osteoarthritis (16\%).

The average MME at time of consult was 93 MME compared with $31 \mathrm{MME}$ at discharge which was statisticially significant $(P<.01)$ (Figure 1$)$. The mean percent change in MME was $46 \%$, including methadone and $42 \%$ excluding methadone. There was a $26 \%$ change in UDS, $28 \%$ change in informed consent, $85 \%$ change in PDMP, 194\% change in naloxone, and $357 \%$ change in STORM reviews from baseline to discharge with all demonstrating statistical significance $(P<.01)$ (Figure 2$)$. At discharge, the most common opioid prescribed was morphine SA (short acting) $(\mathrm{n}=10,13 \%, 44$ average MME) and oxycodone/acetaminophen $(\mathrm{n}=10,13 \%, 28$ average MME) (Table 3).

The average number of days from consult to initial visit was 23 days (Table 4). Faceto-face was the primary means of initial visit with $92 \%(n=69)$ of visits, but phone was the primary mode of follow-up with $73 \%$ of visits $(n=55)$. The average number of follow-up visits was 7, representing 176 average days of time in the Pharmacy Pain Clinic. Consultation to the behavioral health
FIGURE 1 Opioid Dosages for Study Patients

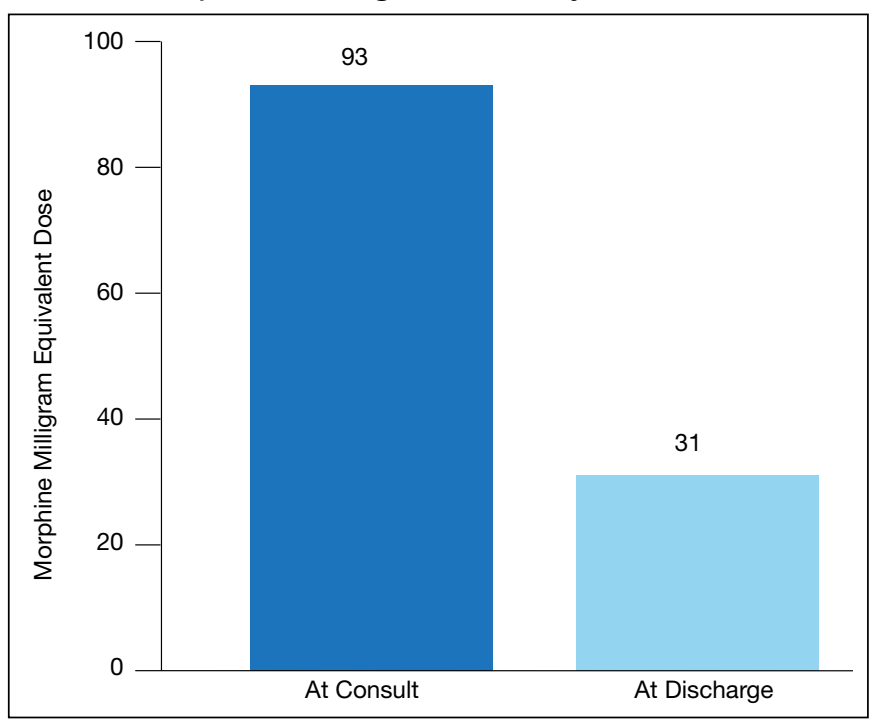

FIGURE 2 Opioid Risk Mitigation Strategy Use

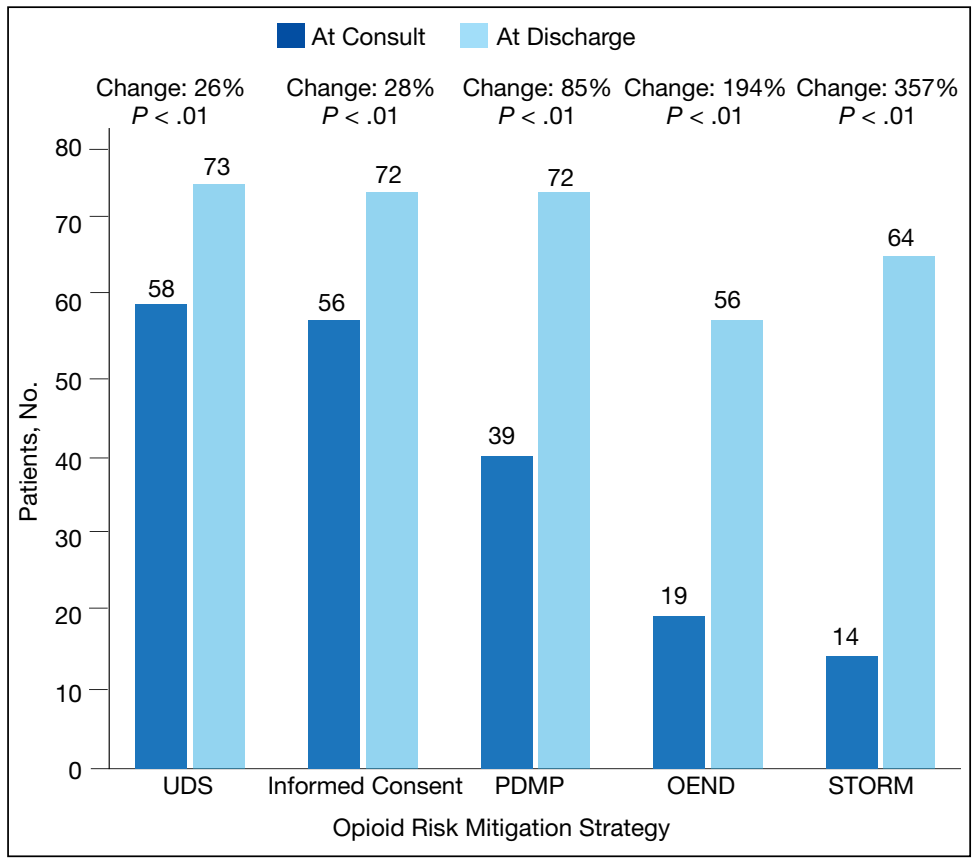

Abbreviations: OEND, Opioid overdose education and naloxone distribution program; PDMP, prescription drug monitoring programs; STORM, stratification tool for opioid risk mitigation; UDS, urine drug screen.

performance program was the most common referral $(n=13,17 \%)$.

Five patients were new opioid starts in the Pharmacy Pain Clinic. Two patients were on tramadol at time of consult. Of the 5 new opioid starts, 3 patients received oxycodone/ acetaminophen, 1 received buprenorphine patch, and 1 received hydrocodone/ 
TABLE 3 Opioid Medications at Consult and Discharge

\begin{tabular}{lll} 
Medications & $\begin{array}{c}\text { At Consult, } \\
\text { No. }\end{array}$ & $\begin{array}{c}\text { At Discharge, } \\
\text { No. }\end{array}$ \\
\hline Buprenorphine buccal film & 1 & 4 \\
\hline Buprenorphine patch & 1 & 7 \\
\hline Codeine/acetaminophen & 1 & 1 \\
\hline Fentanyl patch & 7 & 2 \\
\hline Hydrocodone/acetaminophen & 7 & 4 \\
\hline Hydromorphone extended release & 1 & 0 \\
\hline Hydromorphine immediate release & 2 & 0 \\
\hline Methadone & 20 & 6 \\
\hline Morphine immediate release & 2 & 0 \\
\hline Morphine short-acting & 15 & 10 \\
\hline Oxycodone immediate release & 2 & 4 \\
\hline Oxycodone/acetaminophen & 7 & 10 \\
\hline Oxycodone extended release & 3 & 3 \\
\hline Oxymorphone extended release & 1 & 0 \\
\hline Tapentadol & 3 & 1 \\
\hline Tramadol & 7 & 4 \\
\hline
\end{tabular}

acetaminophen. The new opioid start average was 25 MME. All 5 patients had a UDS for opioid risk mitigation, 4 used consent and STORM reviews, and 2 patients had PDMP checks and naloxone.

\section{DISCUSSION}

There was a statistically significant decrease of the mean MME between the time of consult and the time of discharge. There also were statistically significant changes in use of opioid risk mitigation strategies. Since methadone has a high MME, the mean reduction of MME was calculated with methadone $(46 \%)$ and without methadone (42\%). These data are consistent with other published studies examining opioid tapers in the VA population. Harden and colleagues calculated a $46 \%$ mean reduction in MME over 12 months for 72 veterans from opioid tapers implemented by PCPs, pain service, or pharmacist-run clinics. ${ }^{19}$
There is controversy about equianalgesic doses and no established universal equianalgesic conversion calculator or dose. Numerous equianalgesic opioid dose calculators are available, but for this analysis the CDC MME conversion factors were used (available at: https://www.cdc.gov/drugoverdose /pdf/calculating_total_daily_dose-a.pdf). Previous literature compared existing calculators and found significant variances in calculated doses for methadone and fentanyl conversions. ${ }^{20}$ Additionally, there have been concerns expressed with the safety of the CDC opioid calculator specifically surrounding the conversions for methadone and tapentadol. ${ }^{21}$ In the end, I chose the CDC calculator because it is established, readily available, and consistent.

Pharmacists in pain management can address access issues. ${ }^{2,3,11,12}$ The average length of time from consult to initial visit was 23 days. Often patients may have seen a HCP who implemented a change at the time of consult and wanted the patient to be seen 1 month later. Many patients at the HSTMVH live far from the facility, making in-person visits difficult. A majority of the follow-up visits were conducted by telephone. Patients were offered all modalities available for follow-up, including telephone, in-person, or telemedicine, but patients most often picked telephone. $\mathrm{Pa}$ tients averaged 7 follow-up visits before discharge. This number of visits would have taken time from other health care team members who could have been addressing other veterans. Patients were seen in clinic for 176 days on average, which supports and follows recommendations for a slow, incremental taper.

The opioid medications prescribed changed over time in the clinic. Methadone prescriptions dropped from 20 to 6 at consult to discharge, and fentanyl prescriptions fell from 7 to 2 , respectively. The CDC guideline suggests use of long-acting products with more predictable pharmacokinetics (eg, morphine SA or oxycodone SA) rather than fentanyl or methadone. ${ }^{15}$ Notably, the use of buprenorphine products with FDA approval for pain indications increased from consult to discharge. Many of the patients in this study had pulmonary comorbidities, placing them at higher risk for adverse outcomes. 
Buprenorphine is a partial $\mu$-opioid receptor agonist with a ceiling on respiratory depression so is potentially less risky in those with pulmonary comorbidities.

The biggest changes in opioid risk mitigation occurred in PDMP, OEND program, and STORM reviews. An 85\% increase in PDMP reviews occurred with referral to the clinic. Missouri is the only state without a state-run PDMP. However, the St. Louis County PDMP was developed based on city or county participation and encompasses $85 \%$ of the population of Missouri and 94\% of HCPs in Missouri as of August 29, 2019.22 Because there is no state-level PDMP, a review of the St. Louis County PDMP was not required during the review period. Nevertheless, the Pharmacy Pain Clinic uses the St. Louis County PDMP at the initial visit and regularly during care. VA policy requires a specific note title be used to document each check of the PDMP. ${ }^{23}$

There was a $194 \%$ increase in patients receiving naloxone with consultation to the Pharmacy Pain Clinic. Due to low coprescribing of naloxone for patients prescribed chronic opioid therapy, The author led an interdisciplinary team analysis of health care failure mode effects during the study period. This led to a process change with coprescribing of naloxone at refill in the primary care clinic.

The Comprehensive Addiction and Recovery Act of 2016 mandated that the VA review STORM on new start of opioids or patient identified as "very high-opioid prescription risk" category by an interdisciplinary opioid risk review team. ${ }^{24}$ Thus many of the patients referred to clinic didn't require STORM reviews since they were not new opioid starts or identified as high risk. However, in the standard review of all new patients to the Pharmacy Pain Clinic, a STORM review is conducted and documented to assess the patient's level of risk.

Only 5 patients were started on opioid medications during the study period. This is consistent with both CDC and the joint VA/ US Department of Defense opioid prescribing guidelines that recommend against initiation of opioids for chronic nonmalignant pain. ${ }^{13,15}$ Two of the patients were prescribed tramadol for ineffective pain control at time of consult. Furthermore, 4 of the 5 patients
TABLE 4 Pharmacy Pain Clinic Access, Modes of Care, and Referrals

\begin{tabular}{lc} 
Characteristics & Results \\
\hline Average time from consult to initial visit, d & 23 \\
\hline Mode of initial visit, No. (\%) & $69(92)$ \\
Face-to-face & $6(8)$ \\
Phone & \\
\hline Mode of follow-up visit, No. & 15 \\
Face-to-face & 55 \\
Phone & 4 \\
No follow-up & 1 \\
Both face-to-face and phone equal & 7 \\
\hline No. follow-up visits, mean & 176 \\
\hline Length of treatment in clinic, mean, d & \\
\hline Referrals (Patients may receive $>1)$, No. (\%) & $2(3)$ \\
Acupuncture & $13(17)$ \\
Behavioral health performance program & $1(1)$ \\
Integrative health and wellness & $1(1)$ \\
Occupational therapy & $1(1)$ \\
Neurology & $5(7)$ \\
Pain clinic & $2(1)$ \\
Sleep clinic & $1(1)$ \\
Hospice and palliative care & $1(1)$ \\
Substance use disorder & $1(1)$ \\
Inpatient function restoration program & $1(1)$ \\
Smoking cessation & $52(69)$ \\
None &
\end{tabular}

were started on a short-acting opioid, which was supported by guidelines. ${ }^{13,15}$ One patient was initiated on buprenorphine patches due to comorbid chronic kidney disease. The VA does not limit the quantity of new opioid prescriptions, although some states and private insurance plans are implementing limitations. Guidelines also recommend against exceeding $90 \mathrm{MME}$ due to risk. The average MME in this project at discharge was 25 MME. Use of opioid risk mitigation for the new opioid starts was reasonable. The reason for the missing PDMP report is unknown based on chart review and atypical according to clinic practice.

Recently, efforts to expand pharmacist training and positions in pain management at VA facilities have been undertaken. In 2016, there were just 11 American Society of Health-System Pharmacists-accredited pharmacy postgraduate year 2 pain and palliative care residency programs, which has expanded to 26 sites in $2020 .^{2,3,25}$ In addition, 
the Clinical Pharmacy Practice Office and the VA Office of Rural Health have helped to hire 33 new pain management pharmacists. ${ }^{3}$

The role of pharmacists in prescribing controlled substances is limited mainly due to the small number of states that extend this authority. ${ }^{7}$ At the VA, a pharmacist can practice using any state of licensure. Therefore, a pharmacist working at a VA in a state that does not authorize controlled substance prescribing could obtain a license in a state that does permit it. However, the main barrier to obtaining other state licensures is the cost. At the time the author obtained controlled substance prescriptive authority, little direction was available on the process for advanced practice pharmacists at the VA. Since then, guidance has been developed to ease this process. Educational endeavors at VA have been implemented with the intent to increase the number of pharmacists with controlled substance prescriptive authority.

Barriers to pharmacists providing pain care extend beyond limited controlled substance prescriptive authority. Often pharmacists are still viewed in their traditional and operational role. ${ }^{9,10}$ Other health care team members and patients may not be aware or familiar with the training, knowledge, and skills of pharmacist's and their suitability as an APP. ${ }^{26,27}$ Most states permit pharmacists in establishing CDTA but not all. Additionally, some states recognize pharmacists as HCPs but many more do not. Furthermore, the Social Security Act does not include pharmacists as HCPs. This makes it challenging, though not impossible, for pharmacists to bill for their services. ${ }^{3}$

\section{Strengths and Limitations}

There were numerous strengths of the project. First, this addressed an unmet need in the literature with limited data discussing pharmacist prescribing controlled substances for pain management. There was 1 data reviewer who made the data collection process consistent. Since this retrospectively reviewed controlled substance prescribing in clinic, it captured real-world practice compared with that of experimental models. There were also several limitations in the project. The person collecting the data was also the person who conducted the clinic. The study was conducted retrospectively and based on documented in- formation in the medical record. The population reviewed was primarily male and older, which fits the VA patient population but has less generalizability to other patient populations. This project was conducted at a single VA facility so may not be generalizable to other VA sites. It is unknown whether patients were again prescribed opioids if they left the VA for the community or another VA facility. The pain diagnoses or locations of pain were categorized to main groups and reliant on the referring provider. Another major weakness was the lack of comparison of pain scores or validated objective measure of function at baseline and at discharge. This consideration would be important for future work.

\section{CONCLUSIONS}

Pharmacists functioning as APP are key members of the pain management team. A review of a pharmacy-run pain clinic demonstrated statistically significant reduction in MME and improvement in opioid risk mitigation from consult to discharge. Patients enrolled in the pharmacy-managed clinic also had improvements in adherence to opioid risk mitigation strategies. Future attention should be focused on further expanding training and positions for pharmacists as APP in pain management.

\section{Acknowledgments}

The author thanks Chris Sedgwick for his assistance with data capture.

\section{Author disclosures}

The author reports no actual or potential conflicts of interest with regard to this article.

\section{Disclaimer}

The opinions expressed herein are those of the author and does not necessarily reflect those of Federal Practitioner, Frontline Medical Communications Inc., the US Government, or any of its agencies. This article may discuss unlabeled or investigational use of certain drugs. Please review the complete prescribing information for specific drugs or drug combinations-including indications, contraindications, warnings, and adverse effectsbefore administering pharmacologic therapy to patients.

\section{Disclosures}

Dr. Kominek has received honoraria from Practical Pain Management, PAINWeek, and the American Society of HealthSystem Pharmacists.

\section{References}

1. US Department of Health and Human Services. Help and resources: national opioid crisis. Updated August 30, 2020. Accessed December 10, 2020. https://www.hhs.gov /opioids/about-the-epidemic/hhs-response/index.html

2. Atkinson TJ, Gulum AH, Forkum WG. The future of pain pharmacy: driven by need. Integr Pharm Res Pract. 2016;5:33-42. doi:10.2147/IPRP.S63824 
3. Seckel E, Jorgenson T, McFarland S. Meeting the national need for expertise in pain management with clinical pharmacist advanced practice providers. Jt Comm J Qual Patient Saf. 2019;45(5):387-392. doi:10.1016/j.jcjq.2019.01.002

4. McFarland MS, Groppi J, Ourth $\mathrm{H}$, et al. Establishing a standardized clinical pharmacy practice model within the Veterans Health Administration: evolution of the credentialing and professional practice evaluation process. J Am Coll Clin Pharm. 2018;1(2):113-118. doi:10.1002/jac5.1022

5. US Department of Veterans Affairs, Veterans Health Administration. VHA Handbook. 1108.11. Clinical pharmacy services. Published July 1, 2015. Accessed December 10, 2020. https://www.va.gov/vhapublications/ViewPublication .asp?pub ID=3120

6. US Department of Veterans Affairs, Veterans Health Administration. VHA Handbook 1100.19. Credentialing and priveleging. Published October 15, 2012. Accessed December 10, 2020. https://www.va.gov/vhapublications NiewPublication.asp?pub ID=2910

7. US Department of Justice, Drug Enforcement Agency. Midlevel practitioners authorization by state. Updated February 10, 2020. Accessed December 10, 2020. https://www .deadiversion.usdoj.gov/drugreg/practioners/mlp_by_state .pdf

8. Groppi JA, Ourth H, Morreale AP, Hirsh JM, Wright S. Advancement of clinical pharmacy practice through intervention capture. Am J Health Syst Pharm. 2018;75(12):886-892. doi:10.2146/ajhp170186

9. Chisholm-Burns MA, Spivey CA, Sherwin E, Wheeler J, Hohmeier K. The opioid crisis: origins, trends, policies, and the roles of pharmacists. Am J Health Syst Pharm. 2019;76(7):424-435. doi:10.1093/ajhp/zxy089

10. Compton WM, Jones CM, Stein JB, Wargo EM. Promising roles for pharmacists in addressing the U.S. opioid crisis. Res Social Adm Pharm. 2019;15(8):910-916. doi:10.1016/.sapharm.2017.12.009

11. Hammer KJ, Segal EM, Alwan L, et al. Collaborative practice model for management of pain in patients with cancer. Am J Health Syst Pharm. 2016;73(18):1434-1441. doi:10.2146/ajhp150770

12. Dole EJ, Murawski MM, Adolphe AB, Aragon FD, Hochstadt B. Provision of pain management by a pharmacist with prescribing authority. Am J Health Syst Pharm. 2007;64(1):85-89. doi:10.2146/ajhp060056

13. US Department of Defense, US Department of Veterans Affairs. VA/DoD Clinical Practice Guideline for Opioid Therapy for Chronic Pain. Updated 2017. Accessed November 18, 2020. https://www.healthquality.va.gov/guidelines/Pain/cot NADoDOTCPG022717.pdf

14. US Department of Veterans Affairs. VA, VHA, VA Academic Detailing Service. Veterans Health Administration. Opioid taper decision tool. Updated October 2016. Accessed November 18, 2020. https://www.pbm.va.gov /AcademicDetailingService/Documents/Pain_Opioid
Taper Tool IB 10939 P96820.pdf

15. Dowell D, Haegerich TM, Chou R. CDC guideline for prescribing opioids for chronic pain - United States, 2016 [published correction appears in MMWR Recomm Rep. 2016;65(11):295]. MMWR Recomm Rep. 2016;65(1):1-49. doi:10.15585/mmwr.rr6501e1

16. McPherson M. Demystifying opioid conversion calculations. Published 2009. Accessed November 18, 2020. https://www.ashp.org/-/media/store-files/p1985 -frontmatter.ashx

17. Gudin J, Fudin J, Nalamachu S. Levorphanol use: past, present and future. Postgrad Med. 2016;128(1):46-53. doi:10.1080/00325481.2016.1128308

18. Ogrinc G, Davies L, Goodman D, Batalden P, Davidoff F, Stevens D. SQUIRE 2.0 (Standards for QUality Improvement Reporting Excellence): revised publication guidelines from a detailed consensus process. BMJ Qual Saf. 2016;25(12):986-992. doi:10.1136/bmjqs-2015-004411

19. Harden P, Ahmed S, Ang K, Wiedemer N. Clinical implications of tapering chronic opioids in a veteran population. Pain Med. 2015;16(10):1975-1981. doi:10.1111/pme.12812

20. Shaw K, Fudin J. Evaluation and comparison of online equianalgesic opioid dose conversion calculators. Practical Pain Manag. 2013;13(7):61-66. Accessed November 18, 2020. https://www.practicalpainmanagement.com /treatments/pharmacological/opioids/evaluation -comparison-online-equianalgesic-opioid-dose-conversion

21. Fudin J, Raouf M, Wegrzyn EL, Schatman ME. Safety concerns with the Centers for Disease Control opioid calculator. J Pain Res. 2017;11:1-4. Published 2017 Dec 18. doi:10.2147/JPR.S155444

22. Saint Louis County Public Health. St. Louis County Prescription Drug Monitoring Program. Participating jurisdictions. Accessed December 10, 2020. https:// pdmp-stlcogis.hub.arcgis.com

23. US Department of Veterans Affairs, Veterans Health Administration. VHA Directive 1306: querying state prescription drug monitoring programs. Updated October 21, 2019. Accessed November 18, 2020. https://www.va.gov /vhapublications/ViewPublication.asp?pub_ID=3283

24. Comprehensive Addiction and Recovery Act of 2016. 42 USC § 201 (2016).

25. American Society of Health-System Pharmacists. Residency directory. Accessed November 18, 2020. https:// accreditation.ashp.org/directory/\#/program/residency

26. Feehan M, Durante R, Ruble J, Munger MA. Qualitative interviews regarding pharmacist prescribing in the community setting. Am J Health Syst Pharm. 2016;73(18):14561461. doi:10.2146/ajhp150691

27. Giannitrapani KF, Glassman PA, Vang D, et al. Expanding the role of clinical pharmacists on interdisciplinary primary care teams for chronic pain and opioid management. BMC Fam Pract. 2018;19(1):107. doi:10.1186/s12875-018-0783-9 